\title{
Enhanced RFID Tag Detection Accuracy Using Distributed Antenna Arrays
}

\author{
Ajeck M. Ndifon, Michael J. Crisp, Richard V. Penty, Ian H. White \\ Electrical Division, Department of Engineering, University of Cambridge \\ 9 JJ Thomson Avenue, Cambridge, CB3 0FA, United Kingdom. \\ mn448@cam.ac.uk.
}

\begin{abstract}
An Ultra High Frequency (UHF) Radio Frequency Identification (RFID) system using distributed antenna arrays for interrogating RFID tags in a highly multipath environment is demonstrated. The system makes use of phase diversity and beam steering to overcome fading. The tag detection accuracy is compared to a standard fixed antenna system, showing that the presented system is able to deliver more power to the more challenging tags, and therefore is capable of a higher tag read success rate. It is also shown that, whereas a fixed antenna is capable of scanning a single cell, the ability of a phased array to scan through $360^{\circ}$ azimuth leads to a reduction in number of antennas required for a multicell system. The experimental results are validated using a 3D field-based propagation model, which enables visualisation of the power distribution in the field of interest, and provides insight into the improved system performance.
\end{abstract}

Keywords-RFID; Distributed Antenna System; Phased array antennas; wireless modelling; Ultra High Frequency; Multipath

\section{INTRODUCTION}

The receive power of UHF RFID tags in free space, as with other wireless communication systems, is related to the range by an inverse square law (Friis' equation) [1]. As such, given certain parameters for the reader, antennas and tags, a system could readily be designed and implemented with predictable performance $[2,3]$. In practical environments, however, there are multiple sources of scattering which cause performance degradation due to multipath fading, leading to the presence of nulls/blind spots in the system: points of destructive interference at which a tag's receive power is below its sensitivity threshold, which leads to it not being detected. The situation is exacerbated in wide area RFID systems, in which, multiple antennas and readers are required to provide full coverage of the interrogation zone as shown in Fig. 1. As a result of transmissions from multiple antennas interfering with each other, additional dead zones would be created. Several techniques have been proposed to overcome these problems. The use of time division multiplexing is commonly employed to avoid antenna-antenna interference [4]. Each antenna transmits during a given time slot, and therefore does not interfere with a neighbouring one. As a result, blind spots are avoided. However, this only mitigates multipath due to scattering through spatial diversity. Frequency diversity has also been applied to the problem over the entire 860-960 MHz band [5], but has been shown to be insufficient in the narrow $2 \mathrm{MHz}$ ETSI band allocated for RFID [6]. Phase diversity in a Distributed Antenna System (DAS) has also been shown to improve performance by multicasting the same signal to several distributed antennas, and temporally varying the phase of the signal fed into each antenna, thereby varying the multipath of the channel in order to overcome fading and exploit constructive interference to deliver more power to the tags. [6]. Substantial improvements to the tag detection reliability were achieved with this system. The use of phased array antennas in both the uplink and downlink has also been reported to increase tag read range, as the spatial selectivity of phased arrays allows multiple paths to be minimised or altered, thereby overcoming fading [7-14]. Phased arrays have also been used to obtain localisation of passive tags [15-18]. As a result, several commercial providers have developed phased array RFID antennas with multiple and switched beam configurations [19]. Furthermore, it was shown in [20] that combining multiple phased array antennas with phase diversity has the potential to increase tag power delivery, and therefore detection rate, by multicasting the same signal over two steered beams with phase hopping between them to overcome antenna-antenna interference. It was shown that higher success rates could be achieved compared to the case of time-multiplexing the phased arrays, or multicasting without phase diversity.

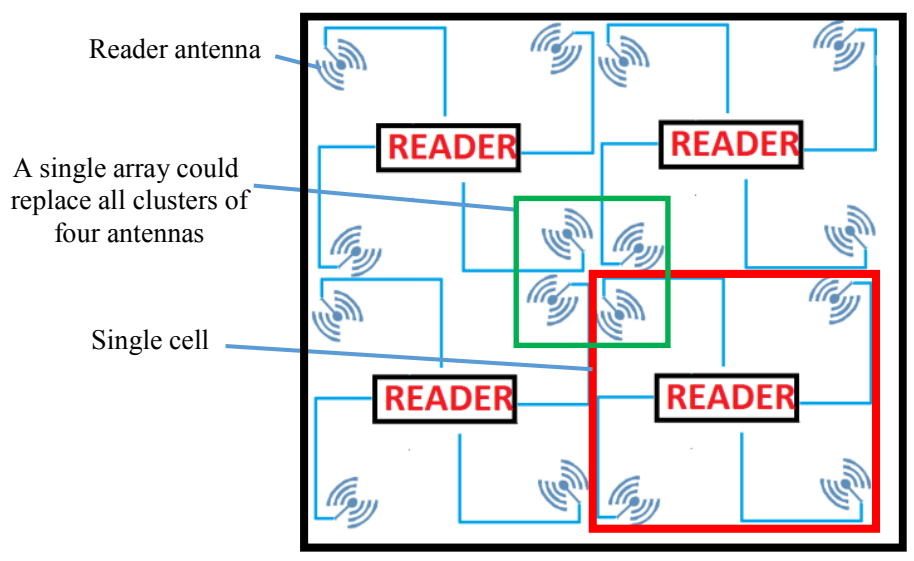

Fig. 1 Typical cellular configuration for a wide area RFID system showing four antennas per cell, and the clustering of four antennas for every four adjacent cells

In this paper, we extend the idea of combined phase diversity and beam steering to a realistic cellular environment, and show that performance improvements could be achieved when compared to a standard fixed antenna configuration. In this light, we demonstrate a distributed system of phased array antennas interrogating RFID tags over a wide area, making use of a combination beam steering and phase diversity between the arrays to increase performance, when compared to a system of fixed antennas. A typical multicell wide area RFID system makes use of a repeating pattern of square cells, with an antenna at each of the vertices as shown in Fig. 1. In the case of a fixed 
antenna system, separate antennas would be required for adjacent cells. However, in the proposed system, each phased array antenna is able to scan a full $360^{\circ}$ in azimuth, thereby allowing a single antenna to be shared by four adjacent cells. If the system with phased arrays can be shown to achieve a similar performance to the fixed antenna system in a single cell, we could reasonably conclude that a four-fold reduction in the number of antennas could be achieved for very large areas (neglecting antennas at the edges of the area). This is because the array can be expected to replicate the same performance in all adjacent cells. We demonstrate that the presented distributed antenna array system outperforms a fixed antenna system in a single cell, thereby satisfying the condition above. In addition, it enables wider cells due to achieving higher read rates for same cell dimensions. Specifically, we compare the performance of a single cell system with four planar $2 \times 2$, phased array antennas to an equivalent system of four fixed antennas. We demonstrate that although the system of fixed antennas delivers more power to the majority of tags, this power is concentrated towards the centre of the interrogation area. The lesser-powered tags at the edges of the cell, receive more power from the system of arrays. This translates experimentally into the overall tag detection accuracy for the proposed array system being higher. Furthermore, we demonstrate that increased performance can be achieved by balancing out the reduction in array gain due to scanning by increasing the transmit power. This performance improvement is achieved by recognising that the gain of the phased array antenna decreases with increased scan angle [21], and can be compensated by increasing the transmit power to reach the legal EIRP limit [22].

The rest of this paper is organised as follows. The second section is devoted to a description of our RFID system architecture. Section III describes the propagation model used, and presents simulations for the different experiments. Section IV describes the antenna system design, as well as the experiments carried out and results analysis. We then conclude in Section V.

\section{SYSTEM DESCRIPTION}

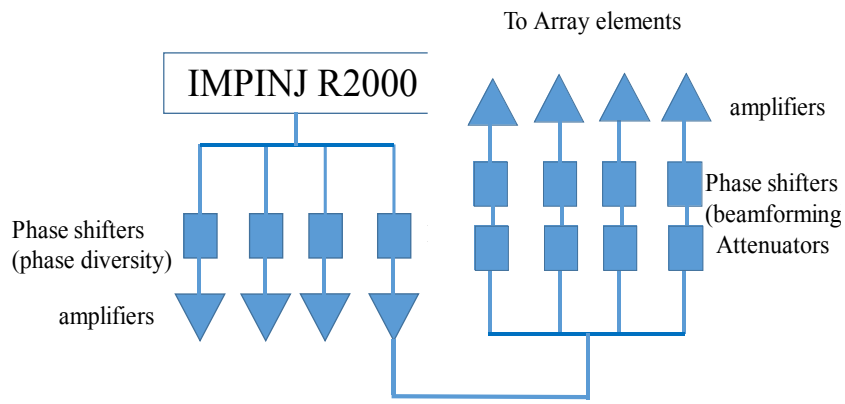

Fig. 2. Architecture of Distributed Antenna Array RFID system showing reader architecture (left), with all of its output ports connected to the feed into the array (only one connection shown, right)

The architecture of the RFID system used in this paper is shown in Fig. 2. An Impinj R2000 System on Chip (SoC) is used to generate the RFID signals, as well as process received tag responses. The output from the SoC is fed into a four-way splitter, and each output is fed into a phase shifter, which is used for maintaining varying phase differences across the antennas to be fed, in order to temporally vary the antenna-antenna inference as described in section I. The signals are then amplified and each fed by coaxial cable into an antenna array or a standard fixed antenna. All beamforming and array processing are performed at the array using a separate PCB. Three separate antennas (not shown) are used to receive the backscatter signals from the tags.

\section{MODELLING AND SimULATION}

Simulations are performed using the 3D model presented in [20]. The model is based on a field interaction between reader and tag antennas. Antennas are modelled using electric field patterns from a full wave Method of Moments (MoM) solver (FEKO). Reader antennas are modelled as circularly polarised (CP) patch antennas, while tags are modelled as dipole antennas. The model also includes a first order Fresnel reflection component from the floor. It is therefore capable of simulating interference patterns resulting from interactions amongst several antennas as well as simple multipath, and takes into account effects such as tag and reader antenna polarisation and alignment. More detail can be found in [20].

A simulation is performed to model the performance of the system proposed, and a comparison is made to a simulated fixed antenna system. A reader antenna is placed at each of the four corners of an $8 \mathrm{~m} \times 8 \mathrm{~m}$ area at a height of $3 \mathrm{~m}$ above the ground level, while tags are placed on the entire plane $1 \mathrm{~m}$ above ground level. Two experiments are performed: one with standard fixed antennas as reader antennas, and the other with $2 \times 2$ antenna arrays as the reader antennas. The fixed antennas are inclined at $45^{\circ}$ to the vertical and face towards the centre of the room (similar to Fig. 1), while the arrays face vertically downward (i.e. they are planar with the ceiling). For each iteration of the simulation, a random phase is applied to each antenna in order to simulate phase diversity as described in Section I. For the case of the arrays, a scan direction $\left(\phi_{0}, \theta_{0}\right)$ is randomly generated, and a beam is generated using equal amplitude and uniform progressive phase excitation:

$$
\boldsymbol{F}(\theta, \phi)=\sum_{n=0}^{N-1} \sum_{m=0}^{M-1} \boldsymbol{E}(\theta, \phi) e^{j k d\left(n \psi_{x}+m \psi_{y}\right)}
$$

where

$$
\psi_{x}=\sin \theta \cos \phi+\phi_{0} \quad \psi_{y}=\sin \theta \sin \phi+\theta_{0}
$$

$\boldsymbol{E}(\theta, \phi)$ is the electric field of a single element antenna obtained from a MoM solver (FEKO), $\boldsymbol{F}(\theta, \phi)$ is the resulting steered beam of the array, $d$ is the array separation distance $(\lambda / 2), k$ is the wave number. In this case $M=N=2$, as we are utilising $2 \times 2$ circularly polarised patch antennas. The simulation is run for 200 iterations and the maximum power for each tag on the plane $1 \mathrm{~m}$ above ground level is recorded. The average value for tags oriented in three orthogonal directions is used. By comparing the maximum power with the tag threshold, we can determine if a tag at that location can be read.

It is seen in Fig. 3 that the fixed antenna system has less power reaching the corners, which happen to be the most critical locations, as the tags here receive the least power, and will 

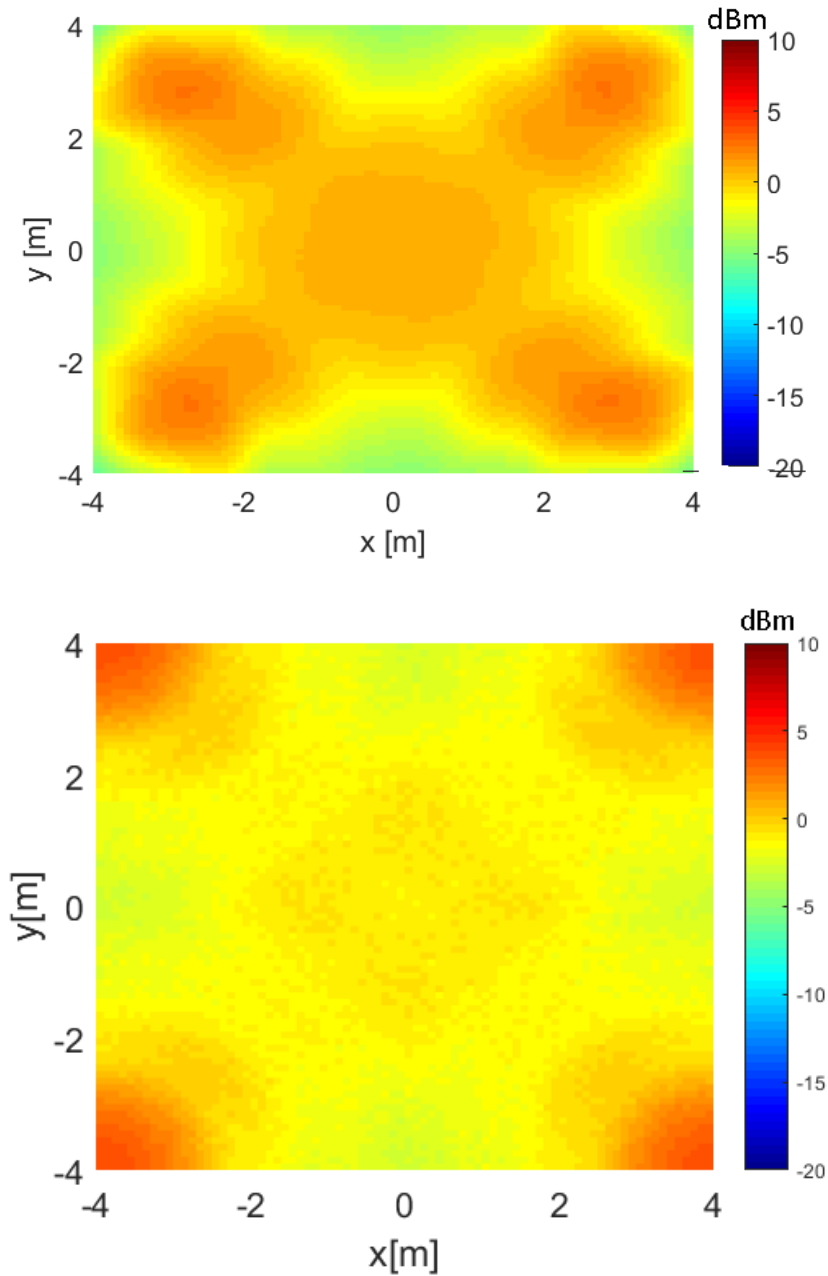

Fig. 4 Average cumulative power distribution in a $8 \mathrm{~m} \times 8 \mathrm{~m}$ room as seen by dipoles (tags) for (a) fixed antenna system with phase diversity (b) array antenna system with beam steering and phase diversity. The power distribution shown is the average of power seen by tags in three orthogonal orientations (i.e. tags oriented Left-Right, Up-Down, and out of the page)

determine the total read success rate. As a result, the power received by tags at these critical locations, which are the more critical tags in determining the read success rate, is greater for the array system. This is demonstrated in a plot of the complementary cumulative probability function (CDF) of the power distribution for both systems in Fig. 4. It is shown that the array system achieves greater power delivery to the tags which receive the least power, and therefore should be capable of achieving a higher tag detection rate.

It should be noted that, according to ETSI regulations [22], if the antenna beamwidth is greater than $90^{\circ}$, the maximum allowed EIRP is reduced by $3 \mathrm{~dB}$. As a result, the antennas at the vertices of the rectangular cell shown in Fig. 1 must be beyond their half power beamwidth. This problem is avoided in the case of an antenna array due to scanning with a beamwidth $<90^{\circ}$. The peak (and mean) power is reduced in the antenna array case, since the array gain decreases with elevation angle. At the optimum $45^{\circ}$ elevation angle [23], it is not possible to obtain the peak gain of the array since array gain decreases with scan angle.

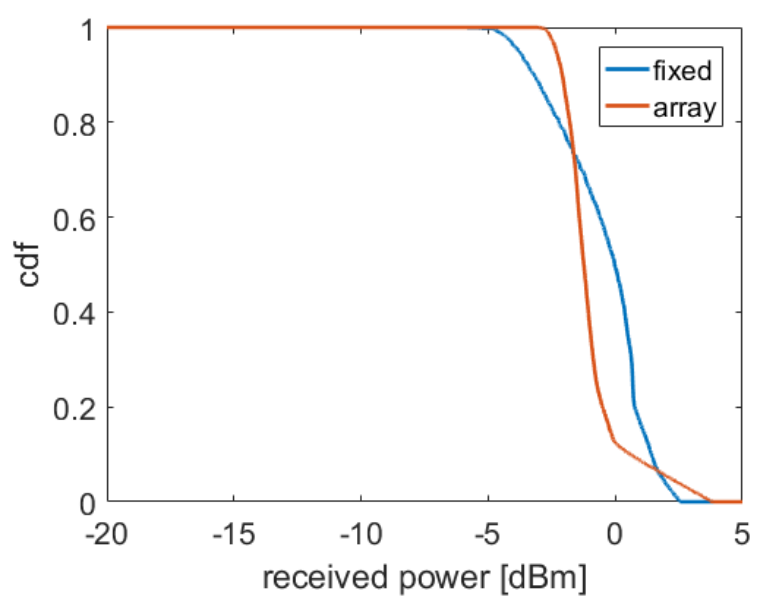

Fig. 3 Simulated cumulative cdf of RF power available to tags for system of (a) fixed antennas and (b) phased array antennas. Plots take into account the average received powe for orthogonally-oriented tags (i.e.

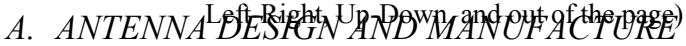

The reader antennas used in the experimental demonstration are circularly polarised microstrip patch antennas. Commercial patch antennas with $9 \mathrm{dBiC}$ gain were used for the fixed antennas. The array antennas were built using ceramic patch elements of dimensions $80 \mathrm{mmx} 80 \mathrm{mmx} 6 \mathrm{~mm}$, with spacings of $173 \mathrm{~mm}(\lambda / 2$ at $865 \mathrm{MHz})$ on an aluminium ground plane as shown in Fig. 5. Mounted on the back is a PCB circuit which performs beamforming. It is equipped with a four-way splitter/combiner for splitting the input RF signal from the reader to feed the four array elements. Each signal is passed through a digital attenuator and phase shifter, which are controlled for beamforming. Processing and control is performed by an onboard Microcontroller Unit (MCU). The four outputs are each fed to a power amplifier, and then to the array elements. The maximum gain of the array is $10 \mathrm{dBiC}$, although the gain decreases with increasing elevation angle from boresight.
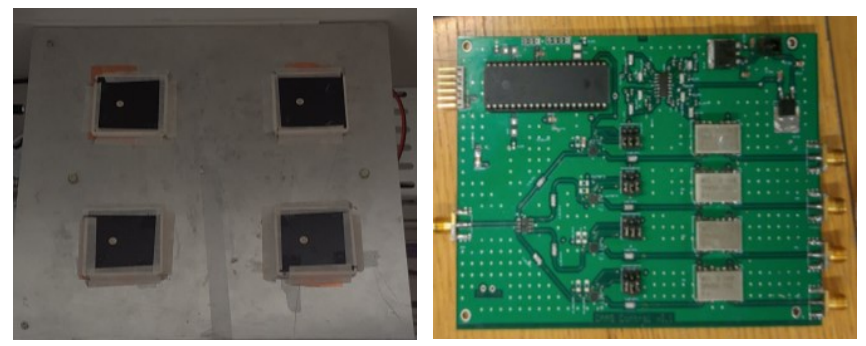

Fig. 5 . (a) $2 \times 2$ antenna array used in experiment. (b) Control PCB with onboard phase shifters and attenuators used for beamforming

B. EXPERIMENTAL SETUP, RESULTS AND ANALYSIS 


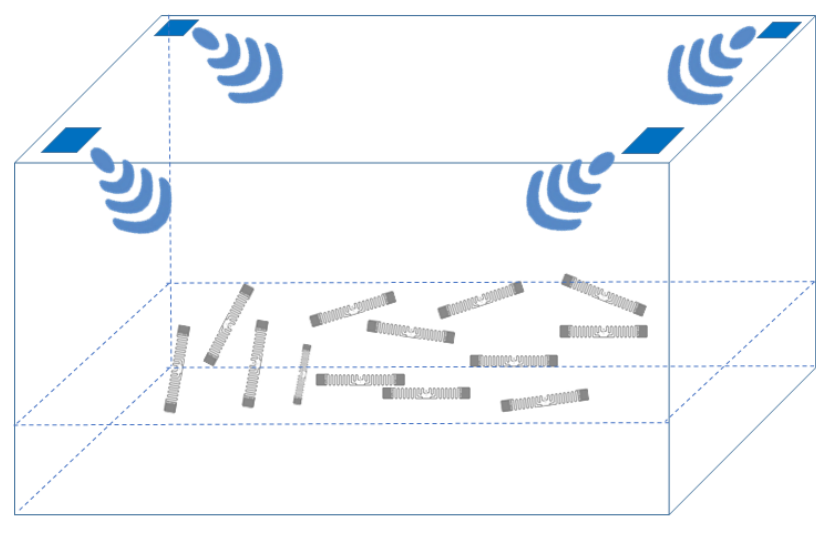

Fig. 6 Representation of experimental work showing antennas at corners of room and distributed tags

The experiment was carried out in an $8 \mathrm{~m} \times 6 \mathrm{~m}$ area (limited by the size of our laboratory) for the two cases presented in the simulations, i.e. four phased array antennas in one experiment, and four fixed antennas in another. In both cases, the antennas are placed at the four corners of the room, with 310 tags of interest distributed in the room. A schematic representation of the experimental setup is shown in Fig. 6. The tags were placed in clusters of 18-40 tags with a roughly uniform distribution over the space. The clustering of tags is for experimental convenience and to provide a more realistic and difficult target case than widely spaced individual tags. A single coaxial cable feeds each array from the reader. A random scan angle for each antenna is generated for every inventory cycle, and the required phases are computed and written to the phase shifter. The required attenuation values are also written to the digital attenuators. Three separate circularly polarised antennas are used as the receive antennas in both cases, to allow comparison of the downlink only. Within the inventory cycle, the relative phase of each array with respect to the other is dithered as in $[6$, 20], and explained in Section I. The experiments matching the simulated scenarios presented above were performed. The CDF of tag RSSI values is plotted in Fig. 7. It is shown that the array achieves a tag detection read success rate of about $99 \%$, while the fixed system achieves $96 \%$. It should be noted however that the experiments are performed using EPC Class 1 Gen 2 session 2 to maximise tag detection within a reasonable period. This session suppresses tags which have been read, from being reread, so the RSSI recorded for each tag is not likely to be close to the maximum from the simulations particularly for those tags with a significant margin above threshold for a variety of antenna combinations. A time evolution of the tag detection rate is also shown in Fig. 8. The read rate of the array system matches that of the fixed system until additional tags are read.

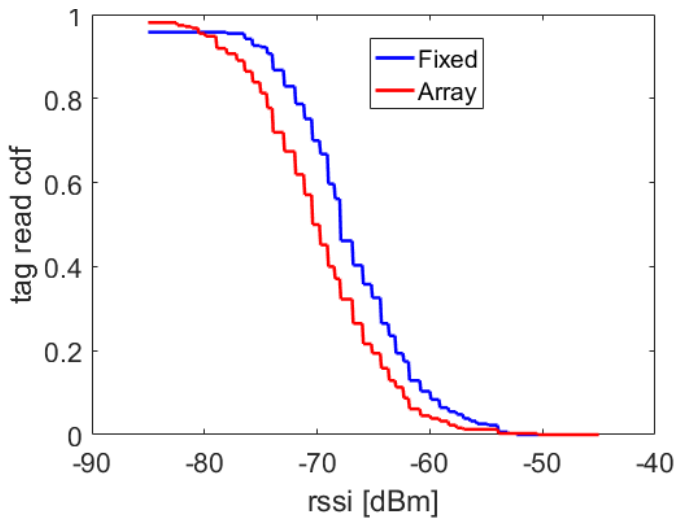

Fig. 7 Cumulative tag read success rate with RSSI for fixed antenna system achieving $96 \%$ after 10 minutes (blue) and antenna array system achieving $99 \%$ (red)

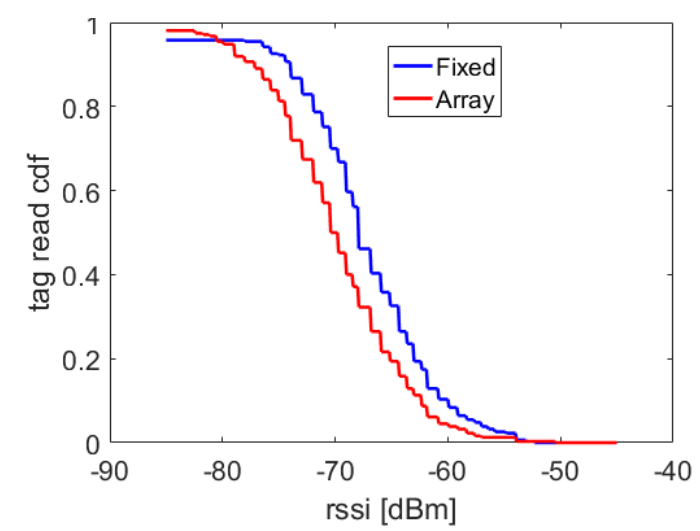

Fig. 8 Cumulative tag read success rate with RSSI for fixed antenna system achieving $96 \%$ after 10 minutes (blue) and antenna array system achieving $99 \%$ (red)

\section{MAXIMUM EIRP BEAM STEERING}

It is well known that the gain of an antenna array decreases with scan angle [21]. This means that to transmit at the maximum allowed EIRP [22], the input power should be modified depending on the scan angle. Fig. 9 shows a plot of gain against both azimuth and elevation scan angles. It should be noted that, due to the directional nature of patch antenna radiation patterns, and the fact that a $2 \times 2$ array is being used, the actual main beam direction differs from the intended scan angle as calculated by equation (1). The plotted requested range of $\theta, \varphi=$ $\pm 150^{\circ}$ corresponds to achieved scan angles of $\pm 90^{\circ}$ in azimuth and $\pm 45^{\circ}$ in elevation. Therefore, beam scanning can be obtained for $360^{\circ}$ in azimuth and up to about $45^{\circ}$ in elevation. It can be seen that, depending on scan direction, the gain can decrease to as low as $7.5 \mathrm{~dB}$, and therefore, a conducted power into the antenna of up to $2.5 \mathrm{~dB}$ more is required for the maximum allowed EIRP at some scan angles. This technique is not able to fully compensate the reduced gain in all directions, since the peak gain cannot be achieved in all directions. 


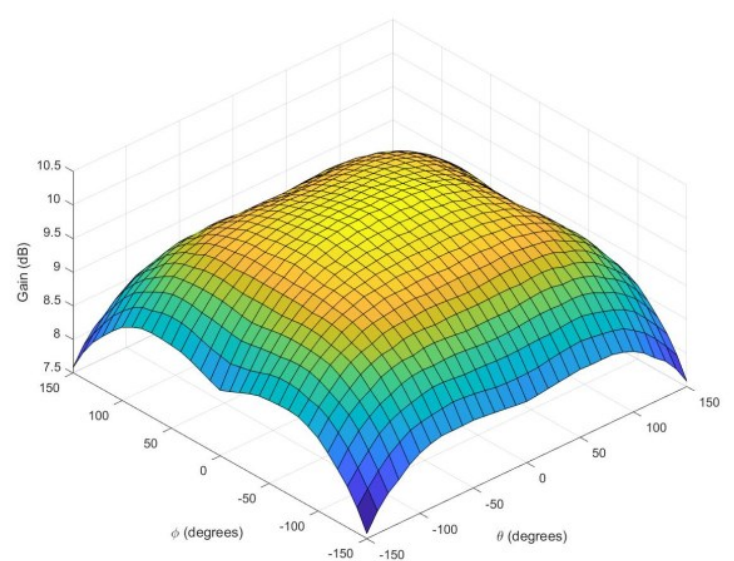

Fig. 9 Maximum array gain degradation with steering angle in both azimuth $(\phi)$ and elevation $(\theta)$. Because of the directional nature of patch antennas, and the limitations of a $2 \times 2$ array, the actual scan angle is significantly lower.

The array processing PCB circuit used in this experiment is equipped with $0.25 \mathrm{~dB}$ step digital variable attenuators to allow the input power to the array to be varied. Depending on scan direction and according to a lookup table of Fig. 9, the attenuation is configured. In this way, we can achieve high effective antenna gain at high elevation angles, and improve the array performance while remaining within EIRP limits. The results of this system are presented in Figs. 10-11. It is shown in this case that a slight increase in tag read rate is achieved, bringing success rate to $100 \%$ in 10 minutes. Again, the modest improvements in RSSI can be explained by the fact that experiments were run in session 2 and represent the RSSI of the first configuration of antenna patterns and phases which result in a received power at the tag above the tag threshold.

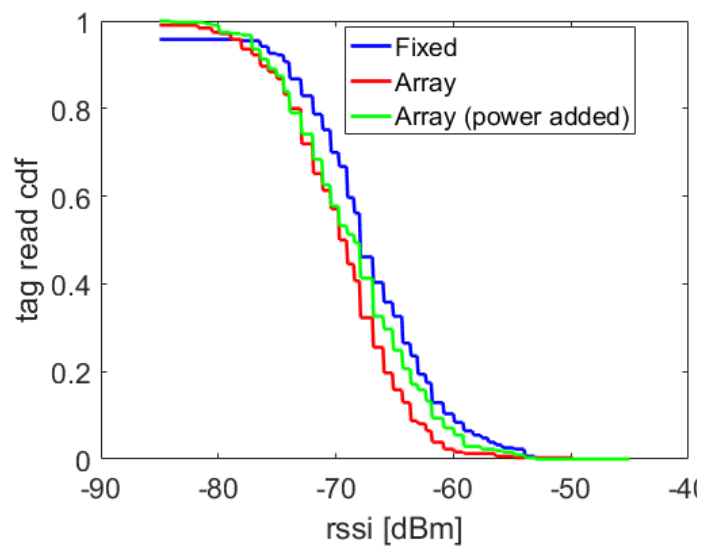

Fig. 10 Cumulative tag read success rate with RSSI for fixed antenna system achieving $96 \%$ after 10 minutes (blue) and antenna array system achieving $99 \%$ (red) and antenna array system with added power achieving $100 \%$ (green)

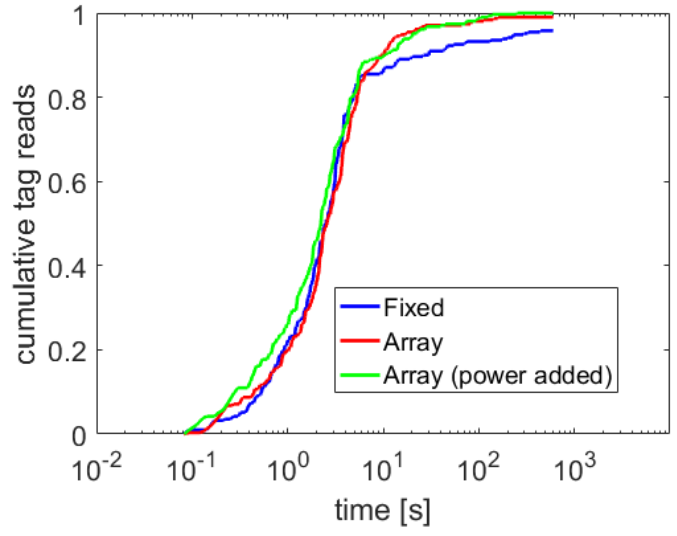

Fig. 11 Cumulative tag read success rate with time for fixed antenna system achieving $96 \%$ after 10 minutes (blue) and antenna array system achieving $99 \%$ (red) and antenna array system with added power achieving $100 \%$ (green)

From the experimental results shown and with reference to Fig. 1 , it can be concluded that, a single $2 \times 2$ phased array antenna can replace the clusters of four antennas in a rectangular multicell RFID system, leading to a reduction in the number of antennas used by a factor of four for a large area, as well as reducing the installation complexity and cost. It has also been demonstrated that, for a single cell the array system outperforms the fixed system in terms of tag detection rate. To further investigate this, the power in the array was reduced by $3 \mathrm{~dB}$. The results, as shown in Fig. 12, demonstrate that a higher detection rate $98 \%$ is obtained at $+30 \mathrm{dBm}$ EIRP compared to $96 \%$ for the fixed system at $+33 \mathrm{dBm}$ EIRP. Since transmit power is proportional to the range squared (Friis' equation), this could be interpreted as at least a $40 \%$ increase in the antenna spacing compared with a fixed system, or a doubling of cell area.

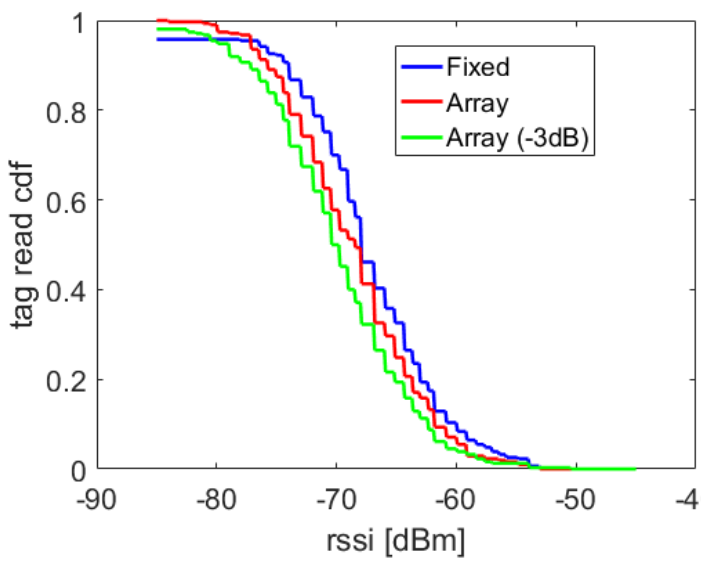

Fig. 12 Cumulative tag read success rate with rssi for fixed antenna system achieving $96 \%$ detection (blue), antenna array system achieving $100 \%$ (red) and antenna array system with $3 \mathrm{~dB}$ less input power $98 \%$ (green) 


\section{THEORETICAL LIMITS}

In this section, we estimate the theoretical limits of this system over a standard system. We assume a pin-like radiation pattern, which is steerable to any direction. It is then implemented in a similar manner to the above system, and we estimate the improvements over a standard antenna system.

Due to the narrow beam width, it will be impractical to do random beam scans due to the time taken. Therefore, a raster scan is implemented and we obtain the time taken to complete a room scan. Phase diversity is also implemented.

\section{CONCLUSION}

An RFID Distributed Antenna Array System has been demonstrated by use of four $2 \times 2$ planar antenna arrays in a $8 \mathrm{~m} \times 6 \mathrm{~m}$ area, and shown to be capable of outperforming a system using 4 conventional antennas with fixed beams in a single cell by delivering more uniform power coverage over the interrogation area, and therefore enabling a higher read accuracy. It is also shown that by increasing the transmit power to compensate for gain reduction due to beam steering, some slight further improvement in performance can be achieved, bringing the detection rate to $100 \%$. It has furthermore been demonstrated that, even with $3 \mathrm{~dB}$ less transmit power, the array system is capable of higher read accuracy than the fixed system, leading to cells potentially $40 \%$ larger (twice the area). Finally, it has been argued and demonstrated that the main advantage in the arrayed system is the potential to scan the entire surrounding area, whereas the fixed antenna system can only be assigned to a fixed cell, thereby leading to a potential reduction in the required number of antennas by a factor of at least four.

\section{REFERENCES}

[1] C. A. Balanis, Antenna Theory: Analysis and Design, WileyInterscience, 2005.

[2] K. V. S. Rao, P. V. Nikitin and S. F. Lam, "Antenna Design for UHF RFID Tags: A Review and a Practical Application," IEEE TRANSACTIONS ON ANTENNAS AND PROPAGATION, vol. 53, no. 12, pp. 3870-3877, 2005.

[3] D. M. Dobkin, "Chapter 6 - Reader Antennas," in The RF in RFID , D. M. Dobkin, Ed., Burlington, Newnes, 2008, pp. 241-303.

[4] M. Yamamoto and T. Yamazoe, "A null-zone control method for RFID systems," in 2011 IEEE Radio and Wireless Symposium, 2011.

[5] C. H. Loo, A. Z. Elsherbeni, F. Yang and D. Kajfez, "Experimental and Simulation Investigation of RFID Blind Spots," Journal of Electromagnetic Waves and Applications, vol. 23, pp. 747-760, 2009.

[6] S. Sabesan, M. J. Crisp, R. V. Penty and I. H. White, "Wide Area Passive UHF RFID System Using Antenna Diversity Combined With Phase and Frequency Hopping," IEEE Transactions on Antennas and Propagation, vol. 62, pp. 878-888, Feb 2014.

[7] M. Abbak and I. Tekin, "RFID Coverage Extension Using MicrostripPatch Antenna Array [Wireless Corner]," IEEE Antennas and Propagation Magazine, vol. 51, pp. 185-191, Feb 2009.

[8] W. S. Lee, S. T. Khang, W. S. Lee and J. W. Yu, "Hemispheric coverage multi-beam switched antenna array using a 4-port feeding network for
UHF RFID dead zone avoidance," in 2013 Asia-Pacific Microwave Conference Proceedings (APMC), 2013.

[9] W. S. Lee, S. T. Khang, W. S. Lee, H. S. Tae and J. W. Yu, "Widecoverage array antenna using a dual-beam switching for UHF RFID applications," in 2013 IEEE International Conference on RFID (RFID), 2013.

[10] L. Weisgerber and A. E. Popugaev, "Multibeam antenna array for RFID applications," in 2013 European Microwave Conference, 2013.

[11] N. C. Karmakar, S. M. Roy and M. S. Ikram, "Development of Smart Antenna for RFID Reader," in 2008 IEEE International Conference on RFID, Las Vegas, 2008.

[12] M. Trinkle and B. Jamali, "ADAPTIVE ANTENNA ARRAYS IN RFID," in Handbook of Smart Antennas for RFID Systems, Online, John Wiley \& Sons, Inc., 2010, pp. 243-281.

[13] M. Trinkle and B. Jamali, "ADAPTIVE ANTENNA ARRAYS IN RFID," in Handbook of Smart Antennas for RFID Systems, Online, John Wiley \& Sons, Inc., 2010, pp. 243-281.

[14] P. Salonen and L. Sydanheimo, "A $2.45 \mathrm{GHz}$ digital beam-forming antenna for RFID reader," in IEEE 55th Vehicular Technology Conference, 2002.

[15] C. Angerer, R. L. r and M. Rupp, "Direction of Arrival Estimation by Phased Arrays in RFID," in European Association for Signal Processing, Aalborg, 2010.

[16] A. Bouzakis and L. Overmeyer, "RFID tag positioning with the aid of an active electronically-steered array," in IEEE 23rd International Symposium on Personal, Indoor and Mobile Radio Communications (PIMRC), Sydney, 2012.

[17] R. Kronberger, T. Knie and R. Leonardi, "UHF RFID localization system based on a phased array antenna," in 2011 IEEE International Symposium on Antennas and Propagation (APSURSI), Spokane, Washington, 2011.

[18] D. Wei, W. Hung and K. L. Wu, "A Real Time RFID Locationing System Using Phased Array Antennas for Warehouse Management," in International Symposium on Antennas and Propagation (APSURSI), Fajardo, 2016.

[19] Impinj, "https://www.impinj.com/platform/connectivity/xarray/," [Online]. [Accessed 10 February 2018].

[20] A. M. Ndifon, M. J. Crisp, R. V. Penty and I. H. White, "Performance improvements of multicast RFID systems using phased array antennas and phase diversity," in IEEE International Conference on RFID Technology \& Application (RFID-TA), Warsaw, 2017.

[21] P. Hannan, "The element-gain paradox for a phased-array antenna," IEEE Transactions on Antennas and Propagation, vol. 12, no. 4, pp. 423-433, July 1964.

[22] GS1, "Regulatory status for using RFID in the EPC Gen2 (860 to 960 $\mathrm{MHz}$ ) band of the UHF spectrum," 2016.

[23] S. Zou, M. Crisp, S. Sabesan, A. Kadri, R. V. Penty and I. H. White, "An optimization model for antenna selection and deployment in single and multi-cell RFID systems," in RFID Technology and Applications (RFID-TA), 2015 IEEE International Conference on, 2015. 